\title{
Retroactive inhibition in rat spatial memory
}

\author{
WILLIAM A. ROBERTS \\ University of Western Ontario, London, Ontario N6A 5C2, Canada
}

\begin{abstract}
Two experiments were carried out in which rats first were given four forced choices on an eight-arm radial maze, then were given interpolated maze experiences, and finally were given a free choice retention test on the first maze. In Experiment 1, interpolated experiences consisted of forced choices made on one, two, or three other mazes, each placed in a different room. Retroactive inhibition (RI) was not found with one and two interpolated mazes but was found with three interpolated mazes. In Experiments $2 a$ and $2 b$, an attempt was made to produce RI within a single context by using two mazes placed side by side or on top of one another and by using interpolated forced choices that were different, random, or the same with respect to forced choices on Maze 1. These conditions failed to yield any evidence of RI. In Experiment 2c, forced choices were followed by interpolated direct placements on the same maze on different, random, or the same maze arms, and retention tests revealed RI under these conditions. It was concluded that rats encode memories of specific places visited in space and that $R I$ will arise only if (1) memory is greatly overloaded with interpolated information or (2) an interpolated visit is made to exactly that position in space to which an animal must travel in order to achieve a correct choice on the retention test.
\end{abstract}

Recent studies of spatial memory in the rat using the radial maze have revealed a surprising excellence of performance (Olton, 1977, 1978, 1979). After freely exploring part of a radial maze or being forced to enter some arms of the maze, rats will choose very accurately only arms not previously visited. Spatial memory appears to be large in capacity, since rats have been found to be able to keep track of visits to as many as 17 (Olton, Collison, \& Werz, 1977) or 32 (Roberts, 1979) places. Also, spatial memory has been found to persist with little loss in accuracy over retention intervals as long as $4 \mathrm{~h}$ (Beatty \& Shavalia, 1980a, 1980b).

It has been suggested also that spatial memory may be impervious to interference. Olton $(1977,1978)$ has tested rats with multiple trials run within a session. When accuracy was plotted as a function of choices within a trial, it was found that nearly errorless performance appeared at the initial choices of each trial. Olton concluded that rats reset their working memory between trials by deleting its contents, and hence each trial in the series was protected from proactive inhibition (PI) from the preceding trials. Roberts and Dale (1981) have pointed out that although rats tested on massed trials do show nearly errorless performance on the initial choices of each trial, accuracy on final choices declines at a progressively faster rate as trials proceed. Furthermore, Roberts and Dale tested rats on repeated trials with a forced-choice

This research was supported by Grant A7894 from the Natural Sciences and Engineering Research Council of Canada. Requests for reprints should be sent to W. A. Roberts, Department of Psychology, University of Western Ontario, London, Ontario N6A SC2, Canada. procedure and found a decline in retention across trials that was more marked at a 60 -sec delay between forced and free choices than at a 0-sec delay. These data indicate PI effects across trials and agree with the well-known finding of a stronger PI effect at a longer retention interval.

In experiments with a retroaction design, Beatty and Shavalia (1980b) and Maki, Brokofsky, and Berg (1979) gave rats four forced choices on an eight-arm radial maze and then subjected them to potentially interfering events prior to a retention test. In the Maki et al. experiments, these events included lights, sounds, a distinctive odor, feeding on the maze, and running a four-arm radial maze in a different place. Beatty and Shavalia used four forced choices on an eight-arm radial maze as interpolated events, with no further testing on the interpolated maze in their first experiment and a final retention test on the interpolated maze in their second experiment. In both experiments, Beatty and Shavalia placed the interpolated events at several different points within a 4-h retention interval. It was found that none of these interpolated experiences caused retroactive inhibition (RI) relative to appropriate control conditions. It is notable that these findings contrast with the findings of short-term memory studies using delayed matching to sample with monkeys and pigeons, in which interpolated changes in illumination produced dramatic losses in retention (Cook, 1980; D'Amato \& O'Neill, 1970; Grant \& Roberts, 1976; Tranberg \& Rilling, 1979).

Some experiments are reported here in which the possibility of RI in rat spatial memory was investigated further. All of these experiments involve extensions and modifications of Experiment 2 in Maki 
et al. (1979) and of the experiments of Beatty and Shavalia (1980b). That is, both experiments reported make use of interpolated experiences on mazes between a series of forced choices and a subsequent free-choice retention test. Retroactive inhibition was found under certain conditions and not under others, and these results may provide some preliminary information for a theory of spatial memory encoding in the rat.

\section{EXPERIMENT 1}

In both Maki et al.'s (1979) second experiment and Beatty and Shavalia's (1980b) experiments, rats were given interpolated runs in a place different from that in which initial forced choices and the retention test were given. Both areas were reported to be rich in extramaze cues, which served to differentiate the two contexts. Both Chiszar and Spear (1969) and Zentail (1970) have demonstrated that the use of differentiated contexts for original and interpolated learning leads to reduction of RI for retention of spatial habits, relative to habits learned in the same context. Therefore, we might expect that runs given on radial mazes in similar contexts might yield evidence of RI. In Experiment 1, rats were given interpolated runs on mazes placed in rooms highly similar to that in which the initial maze was run.

A second factor examined in Experiment 1 was the number of interpolated mazes. Earlier studies of maze learning in rats attempted to find evidence of RI using multiple-choice mazes. Ho (1928) and Waters and Vitale (1945) trained rats to accurately traverse an initial maze and then gave different groups of animals different numbers of trials on a new maze. Relearning tests on the original maze showed no effect of interpolated learning. However, Marx (1944) gave rats interpolated learning on 0, 1, 4,8 , or 12 different water mazes after learning an initial water maze. Relearning tests on the initial maze showed a growth in RI to a maximum at 4 and 8 interpolated mazes, with some decline in the effect at 12 mazes. Since number of interpolated mazes may be a critical factor, and Beatty and Shavalia and Maki et al. used only one interpolated maze, rats in Experiment 1 were tested with one, two, and three interpolated mazes. Although a single interpolated maze might not cause RI, learning two or three interpolated mazes might overload memory and produce RI.

\section{Method}

Subjects. The subjects were 10 male hooded rats that were between 120 and 140 days of age at the beginning of the experiment. Prior to preliminary training, body weights were reduced to $85 \%$ of free-feeding weights.

Apparatus. Four identical eight-arm radial mazes were constructed of unpainted plywood. Each maze consisted of a central octagon, $30 \mathrm{~cm}$ in diameter, and eight identical arms, $7.5 \mathrm{~cm}$ wide $\times 48.5 \mathrm{~cm}$ long. Each arm contained $10.3-\mathrm{cm}$-high walls along its length, and holes $2 \mathrm{~cm}$ in diameter and $.8 \mathrm{~cm}$ deep were drilled at the end of each arm to act as food wells. Adjacent to the central platform, the walls of each alley contained runners for a guillotine door, which could be easily slid into place to block entrance into the alley. The mazes were supported on legs, which raised them $60 \mathrm{~cm}$ above the floor.

The experiment was carried out in four rooms, each $3.1 \mathrm{~m}$ long $\times 2.3 \mathrm{~m}$ wide, that were adjacent to one another along a corridor. These rooms were highly similar to one another in that each was painted pale green and each contained a table, two chairs, and a wastepaper basket. Illumination was provided in each room by centrally placed in-ceiling fluorescent lights, covered by a plastic diffuser. The rooms were differentiated somewhat, however, in that Rooms 1 and 3 were not perfectly rectangular, with protrusions along one wall of each, whereas Rooms 2 and 4 were exact rectangles. Also, the furniture was arranged somewhat differently from one room to the next. Each room contained one eight-arm radial maze.

Procedure. During an initial 4 days of pretraining, each subject was allowed to explore each of the mazes freely for about $15 \mathrm{~min}$ per day, with several $45-\mathrm{mg}$ food pellets placed along the ends of the arms. Once animals were exploring the mazes freely and consuming the pellets, 4 more days of preliminary training were given in which one pellet was placed in the food well at the end of each arm (the standard reward throughout all experiments), and each animal was aliowed to run one maze on each day until all eight arms had been eniered. Each rat was trained with a different maze on each of these 4 days.

At the completion of preliminary training, Experiment 1 was carried out over a period of 24 days. Each subject was tested repeatedly on six different conditions. Three of these conditions were $\mathbf{R I}$ conditions, in which rats were forced to enter four alleys on Maze 1 in Room 1 and then were required to make forced alley entrances on one, two, or three further interpolated mazes in Rooms 2, 3, or 4. After the final interpolated maze, the animal was returned to Room 1 and tested for retention of the alleys originally entered on Maze 1. In addition, retention tests were given for the forced entrances on interpolated mazes. The other three conditions were control conditions for the interpolated-maze conditions. On control trials, animals made forced alley entrances on Maze 1 and then were detained for the length of time it took to run one, two, or three interpolated mazes before being tested for retention of Maze 1. Each of these RI and control conditions was tested once within each of four blocks of 6 days. The RI conditions always were tested on odd-numbered days, and the control conditions always were tested on even-numbered days.

On RI test trials, all eight arms of Maze 1 were baited with one pellet, and four randomly chosen arms were blocked off with guillotine doors. The subject then was placed on the center platform and allowed to enter the four open arms and eat the pellet at the end of each one. As soon as the animal had returned to the center platform after entering all four arms, it was removed from the maze and taken to the maze in Room 2 . The second maze also had all eight arms baited and four randomly chosen arms blocked off. The rat was placed on Maze 2 and allowed to collect all four pellets available. In conditions that called for two or three interpolated mazes, the rat was taken from Room 2 and given further forced choices on mazes in Rooms 3 and 4. When the final interpolated maze had been completed, the subject was taken back to Room 1 and placed on its maze with all eight alleys open but only the alleys previously blocked baited with food. Free choices were allowed until the subject had collected the four remaining pellets. The rat then was taken to Room 2 and allowed to choose freely among all eight arms on Maze 2 until all remaining pellets had been found, and this procedure was repeated for any remaining interpolated mazes.

Throughout the RI trials, the times taken to complete interpolated trials were recorded. These times were used as the retention interval on control trials. Thus, the retention interval on control trials for one, two, and three interpolated mazes was the length of 
time taken to complete one, two, or three interpolated mazes. Each animal's control retention intervals were based on its own speed of running interpolated mazes. Since these times declined over days of testing, each subject's retention interval on control trials was based on its most recently tested RI trials. During the retention interval, the rat was placed in a cage in Room 1 with a dish containing the number of food pellets that it would find on interpolated mazes on the appropriate RI condition. The subject was removed from the cage at the end of the retention interval and allowed to choose freely among all eight arms on Maze 1 until all four of the remaining pellets had been collected.

\section{Results}

Over the last 4 days of preliminary training, rats showed an increasing tendency to enter arms not previously visited. The mean numbers of different arms entered on the first eight choices were 6.5, 6.9, 6.8, and 7.2 on Days 1, 2,3, and 4, respectively.

The results of the RI test trials are shown in Figure 1 . The dependent variable is the mean percentage of correct choices made on the first four alley entrances of the retention test. Accuracy is plotted separately for each of the RI conditions, in which animals were given forced choices followed by retention tests on two, three, or four mazes. The independent variable is the ordinal position of the maze tested within a trial. It should be noted that retention of all mazes after the first is susceptible to both RI and PI influences. The input or forced-choice phase of each trial on Maze 2 was preceded by input on Maze 1, input on Maze 3 was preceded by input on Mazes 1 and 2 , and input on Maze 4 was preceded by input on

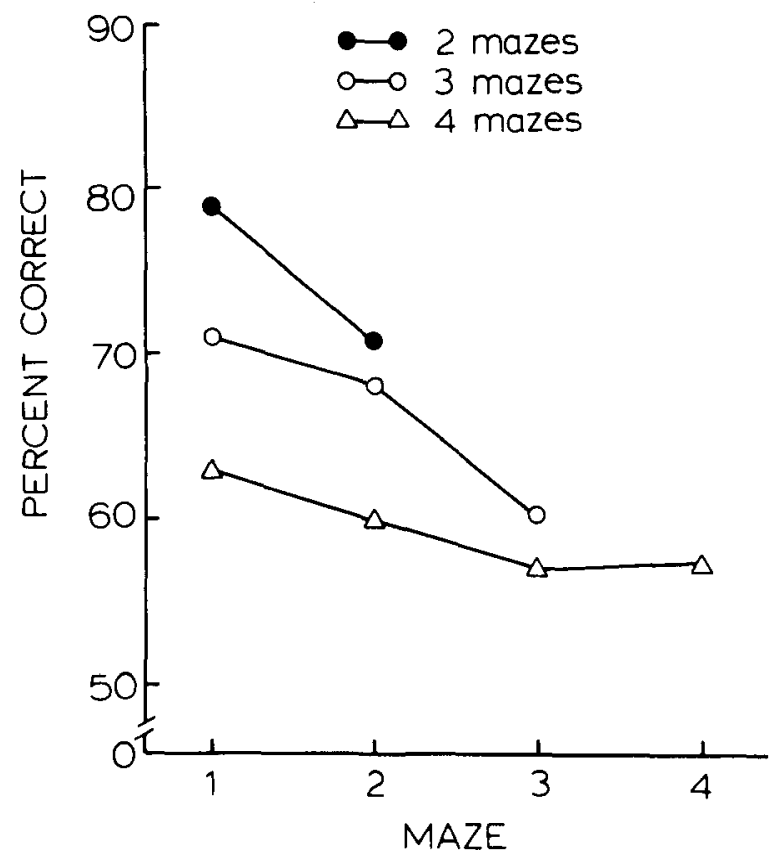

Figure 1. Percentage of correct responses made on the first four free choices plotted as a function of the ordinal position of the maze tested. Separate curves are presented for conditions in which rats were given forced cholces on two, three, and four mazes.
Mazes 1, 2, and 3. In addition, as retention tests proceed within a sequence, potential output interference from previous retention tests arises. Retention tests on Mazes 2, 3, and 4 were preceded by output or retention tests on 1, 2 or 3 mazes, respectively. If increases in the number of sources of input and output interference create increasingly stronger RI and PI, we would expect that performance would decline both as the number of mazes tested increases and as the ordinal position of mazes tested within a trial increases. The data presented in Figure 1 suggest this trend.

Several analyses of variance were performed on the data shown in Figure 1. Performance on Maze 1 decreased significantly as the number of input mazes increased from two to three to four $[F(2,18)=10.67$, $\mathrm{p}<.011$. The data from tests on Mazes 1 and 2 were used to perform a test position ( 1 and 2$) \times$ number of mazes input $(2,3$, or 4$) \times$ subjects analysis, and it was found that accuracy declined as the number of mazes input increased $[F(2,18)=10.63, p<.01]$; no significant effects of test position $[F(1,9)=3.33$, $p>$ $.05]$ or of the interaction of number of mazes input and test position ( $F<1$ ) were found. By using only the lower two curves in Figure 1, the same type of analysis was performed with Test Positions 1,2 , and 3 and number of mazes input set at three and four. This analysis revealed significant effects of test position $[F(2,18)=8.98, p<.01]$ and of mazes input $[F(1,9)=6.75, p<.05]$ but not of the interaction of these factors $(F<1)$. A final test position $\times$ subjects analysis was performed on the four-mazes curve in Figure 1 and yielded a nonsignificant effect of test position $[F(3,27)=2.68, p>.05]$.

In order to determine whether significant retention was displayed in each of the conditions shown in Figure 1 , each point was tested against $50 \%$. Since four of the eight arms on the maze were designated as correct, and rats very rarely entered the same arm twice on the first four free choices, sampling without replacement was assumed on the free choice test, and $50 \%$ was set as the level of accuracy expected by chance. All nine of the points seen in Figure 1 were found to be significantly above the $50 \%$ level by $t$ test $(p<.05)$. Therefore, some retention was manifested even in the conditions that experienced the greatest interference.

Although the data presented in Figure 1 suggest that decreases in accuracy arise from increasing sources of input and output interference, the retention interval increased as number of mazes input or number of output tests increased. Only in the case of retention tests on Maze 1 with one, two, or three interpolated mazes were control trials run for the passage of the retention interval. In Figure 2, the percentage of correct choices on Maze 1 is plotted as a function of the number of interpolated mazes for the 


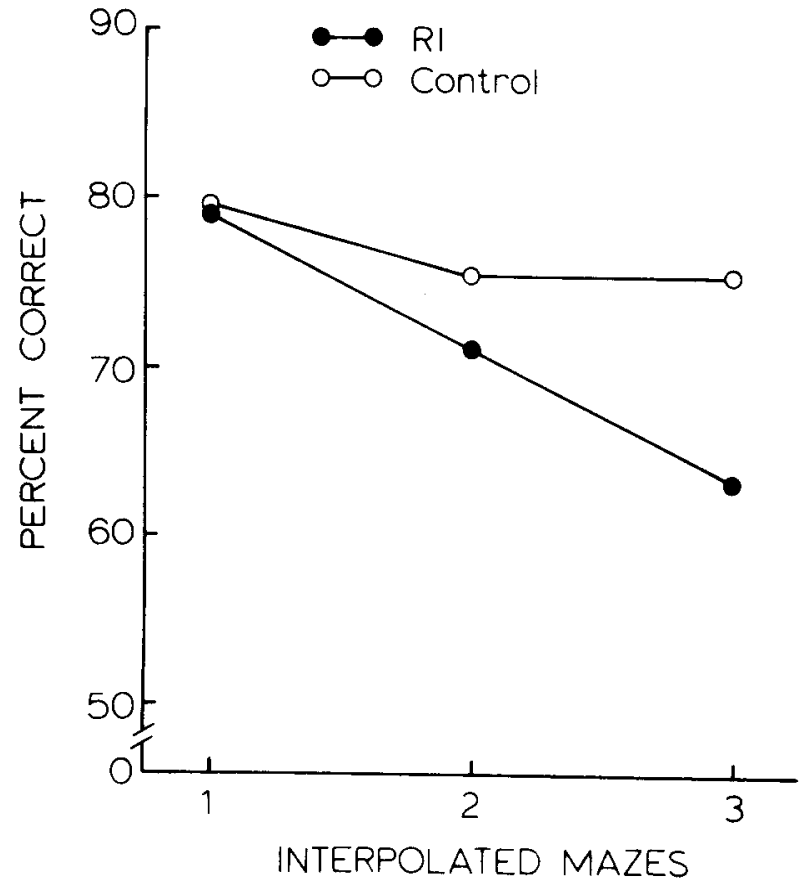

Figure 2. Percentage of correct responses made on the first four free choices of Maze 1 plotted as a function of interpolated mazes for the retroactive Inhibltion (RI) condition and as a function of the time required to complete interpolated mazes for the control condition.

$\mathrm{RI}$ condition and as a function of the interval taken to run the interpolated mazes for the control condition. The overall mean retention intervals were 58.0, 129.7 , and $227.8 \mathrm{sec}$ with one, two, and three interpolated mazes, respectively. These data were analyzed within an analysis of variance containing RI vs. control conditions, interpolated mazes, and subjects as factors. The interpolated mazes factor was significant $[F(2,18)=4.24, p<.05]$, but nonsignificant effects were found for the RI vs. control conditions factor $[F(1,9)=3.60, p>.05]$ and the interaction of interpolated mazes $\times$ RI vs. control $[F(2,18)=2.04$, $p>$.05]. Subsequent analyses showed that control performance did not vary as a function of the retention interval $(F<1)$, but, as already reported for the Maze 1 points in Figure 1, performance declined significantly as a function of interpolated mazes in the RI condition. Comparisons of RI vs. control performance at each level of interpolated mazes indicated no significant differences with one $(t<1)$ or two $[t(9)=1.48, p>.05]$ mazes but a significant $R I$ effect with three interpolated mazes $[\mathrm{t}(9)=3.50, \mathrm{p}<.01]$.

\section{Discussion}

These findings suggest that RI can be obtained in spatial memory tests with rats under certain conditions. These conditions are the presentation of a large amount of interpolated experience on identical mazes in similar environments. The findings of Beatty and Shavalia (1980b) and Maki et al. (1979) were replicated here in that requiring rats to run one interpolated maze caused no RI. It was only when three interpolated mazes were used that significant RI appeared. The results of retention tests given on mazes after the first one tested suggest that further interference with retention may have arisen from PI and output interference effects, but appropriate controls for the passage of time were not included in the experiment.

\section{Experiment 2}

Although Experiment 1 succeeded in demonstrating RI with rather massive interpolated input, rats still showed a surprising resistance to RI at one and two interpolated mazes. The rooms in which Maze 1 and interpolated mazes were encountered were similar but not identical. It is possible that rats were very sensitive to this contextual change and were able to discriminate well among rooms. Such discrimination might allow animals to keep experiences on different mazes separate in memory, at least up to a certain load capacity.

Retroactive inhibition has been demonstrated in rats when two conflicting maze habits have been sequentially learned within the same context. If rats are trained to go to one side of a T-maze until this habit is established and then are trained to go to the opposite side of the same maze, strong RI is found when the first habit is relearned (Chiszar \& Spear, 1969; Crowder, 1967; Frankmann, 1957; Zentall, 1970).

Experiment 2 consisted of a set of three related experiments, in which rats were tested for RI with mazes placed within the same context. In Experiment $2 \mathrm{a}$, two mazes were placed side by side in the same room, and, in Experiment $2 b$, one maze was placed on top of the other. Rats were given forced choices on one maze followed by forced choices on the other maze and then were brought back to the first maze for a retention test. In Experiment 2c, a single maze was used and direct placements on selected arms were used as interpolated input. Since a good deal of evidence suggests that rats make substantial use of extramaze visual cues in running the radial maze (Olton, 1978; Roberts, 1979), the use of two mazes within the same environment with arms pointing toward similar sets of extramaze cues may cause RI. This might be particularly true if rats code arms entered in terms of gross environmental features seen from the ends of each arm and the input on the initial maze conflicts with that on the interpolated maze.

In all three experiments, an experimental design was used that maximized sensitivity to any possible confusion between arms entered on adjacent mazes. 
In Experiments $2 \mathrm{a}$ and $2 \mathrm{~b}$, mazes were oriented so that for each arm of one maze, there was a corresponding arm of the other maze that pointed in exactly the same direction. Arms pointing in the same compass directions were given the same numbers on each maze. Four conditions were tested-different, random, same, and control. Suppose that a rat was forced to enter Arms 2, 3, 5, and 8 on Maze 1. In the "different" condition, the rat was forced to enter Arms 1, 4, 6, and 7 on the interpolated maze. In the "random" condition, the choice of alleys to be entered on the interpolated maze was random relative to forced choices on Maze 1; the animal might be forced to enter Alleys 2, 4, 7, and 8 on the interpolated maze in the "random" condition. Under the "same" condition, Arms 2, 3, 5, and 8 would be entered on the interpolated maze. The control condition involved the omission of runs on the interpolated maze; rats remained idle for the length of time taken to run the interpolated maze and then were tested on Maze 1.

If rats confuse arms pointing in the same directions on different mazes, we would expect very strong RI in the "different" condition, relative to the control condition. Substantial RI in the "random" condition would be expected also, although perhaps not as strong as that found with the "different" condition. By way of contrast, we might expect that the "same" condition would show retroactive facilitation or better performance in the "same" condition than in the control condition. This could arise if runs in the same directions on both mazes acted to reinforce memory of the arms entered on Maze 1. It seems clear from this theoretical analysis that the greatest difference should appear between the "same" and "different" conditions.

\section{Experiment 2a}

\section{Method}

Subjects. The rats used in Experiment 1 were used again as subjects in Experiment 2a.

Apparatus. Two of the mazes used in Experiment 1 were placed side by side in Room 1, with the nearest arms separated by $30 \mathrm{~cm}$. The mazes were aligned so that Arm 1 on both mazes pointed in the same direction and hence the corresponding pairs of Arms 2-8 on each maze pointed in the same directions.

Procedure. The experiment was carried out over a period of 12 days, which consisted of three blocks of 4 days each. Within each 4-day block, each animal was tested once on each of the "different," "random," "same," and control conditions, with the order in which conditions were tested varying randomly between animals and blocks of days.

One maze was designated as Maze 1, and the other maze was designated as the interpolated maze throughout the experiment. Each trial was initiated by baiting all arms of Maze 1 and blocking off the arms to four randomly chosen alleys. The rat then was placed on the central platform and allowed to enter all four open arms and collect the pellet at the end of each. In the interpolated maze conditions, the subject then was taken off Maze 1 and placed on the interpolated maze, with four alleys blocked in such a way as
Table 1

Percentage of Correct Choices Made on Different, Random, Same, and Control Conditions in Experiments $2 \mathrm{a}, 2 \mathrm{~b}$, and $2 \mathrm{c}$

\begin{tabular}{ccccc} 
& \multicolumn{4}{c}{ Condition } \\
\cline { 2 - 5 } Experiment & Different & Random & Same & Control \\
\hline 2a & 85.8 & 86.7 & 91.7 & 85.0 \\
2b & 77.5 & 76.9 & 84.4 & 78.1 \\
2c & 72.5 & 77.5 & 86.2 & 83.8 \\
\hline
\end{tabular}

to allow the subject to enter arms pointing in different, same, or random directions with respect to the arms entered on Maze 1. One pellet was available at the end of each of the four arms entered on the interpolated maze. When an animal had returned to the central platform after entering all four open arms on the interpolated maze, it was returned to Maze 1 for a retention test. All eight arms were open, but only the four arms not entered on the original run contained a food pellet. The trial was complete when all four food pellets had been collected. When the control condition was tested, the animal was allowed to make four forced choices on Maze 1 and then was placed in a cage in the same room as the mazes and allowed to eat four food pellets. The retention interval spent in the cage corresponded to the time most recently taken by that animal to complete the interpolated maze. At the end of the retention interval, the subject was returned to Maze 1 for the standard retention test.

\section{Results}

The results are shown in the first row of Table 1 , in which the percentage of correct responses on the first four choices of the retention test are presented for "different," "random," "same," and control conditions. The mean retention interval for the control condition was $75.3 \mathrm{sec}$. The data indicate very little difference in accuracy between the "different," "random," and control conditions, with the "same" condition being somewhat higher than the others. A conditions $\times$ subjects analysis of variance yielded a nonsignificant effect of conditions $[F(3,27)=1.40$, p > .05]. Furthermore, a Newman-Keuls test comparing all four means with one another showed no significant differences for any pair of conditions.

These findings offer no support for the hypothesis that RI would be caused by confusion between arms entered on separate mazes in the same context, with corresponding arms pointing toward the same extramaze cues. Rather, these findings suggest the possibility that rats can discriminate well between memories established on separate mazes in the same context, as long as those mazes are in separate places.

\section{Experiment 2b}

No evidence of RI was found in Experiment $2 a$ when mazes were displaced horizontally. In Experiment $2 b$, two mazes were placed at exactly the same floor position within a room but were displaced from one another vertically. In other words, one maze was placed directly above the other, with the correspond- 
ing arms of each pointing in exactly the same directions. "Different," "random," "same," and control conditions were tested once again. This experiment examines the possibility that RI will occur when memories are formed on two mazes that differ only in the height from which extramaze cues are viewed.

\section{Method}

The subjects were the same 10 rats used in the preceding two experiments. Two of the mazes used in Experiment 1 were placed on top of one another. This was accomplished by placing small pieces of wood across the alleys of the bottom maze, so that the legs of the top maze could rest on them. Thus, the bottom maze was elevated $60 \mathrm{~cm}$ above the floor, and the top maze was raised another $60 \mathrm{~cm}$ above the alleys of the bottom maze. Alleys $1-8$ on each maze pointed in exactly the same compass directions within the room.

The experiment was carried out for a period of 16 days, or four blocks of 4 days each. Within each 4-day block, "different," "random," "same," and control conditions were each tested once, with the order of testing conditions randomized between subjects and blocks of days. For five subjects, the bottom maze was Maze 1 and the top maze was the interpolated maze on the first and third blocks of days; on the second and fourth blocks of days, the top maze was Maze 1 and the bottom maze was the interpolated maze. This assignment of mazes to blocks of days was reversed for the remaining five subjects. The alleys entered on Maze 1 and the interpolated maze within the four conditions tested were determined in exactly the same manner as used in Experiment $2 \mathbf{a}$.

\section{Results}

The second row in Table 1 displays the mean percentages of correct choices for the retention tests given under "different," "random," "same," and control conditions. The pattern of results is very similar to that found in Experiment 2a: The "different," "random," and control conditions appear to differ little from one another, and the "same" condition demonstrates modestly better performance. The mean retention interval for the control condition was $68.6 \mathrm{sec}$. Statistical analysis showed that conditions of testing was not a significant factor $[F(3,27)=1.03$, p > .05], and a Newman-Keuls test failed to show a significant difference between any condition and any other condition. Subjects scored slightly better when tested on the bottom maze (81.2\%) than when tested on the top maze $(\mathbf{7 7 . 2 \%})$, but there were no significant effects of maze $[F(1,9)=2.12, p>.05]$ or of the conditions $\times$ maze interaction $(F<1)$.

These results, taken in conjunction with those of Experiment $2 a$, indicate that rats apparently can discriminate quite accurately between memories established on two mazes within the same context. Furthermore, this discrimination occurs even when the arms of the mazes point in exactly the same directions and alleys entered on the interpolated maze are designed to create maximal interference on the retention test ("different" condition). Interference appears to be minimized as long as mazes are separated somewhat in space, either horizontally or vertically.

\section{Experiment 2c}

The assumption has been made that animals failed to show RI in Experiments $2 a$ and $2 b$ because Maze 1 and the interpolated maze were spatially separated. However, this assumption has not been proved. Would RI appear if Maze 1 and the interpolated maze were the same? In Experiment $2 c$, this question is addressed by using a single maze. Animals were forced to enter four randomly selected arms as the initial learning experience and then were placed directly on the ends of four arms and allowed to eat a pellet placed there. The arms on which animals were placed had a different, random, or same relationship to the arms previously entered. In the control condition, animals were detained for the length of time taken for the four arm placements.

Walker and Olton (1979) have shown that rats placed on selected arms of a four-arm radial maze will avoid entering those arms when subsequently given a free choice test. If simply being placed at a position in space allows an animal to code that position as a place visited and not one to be returned to immediately, then the "different" and "random" conditions should show RI relative to the "same" and control conditions, since the alleys that must be entered in the former conditions in order to demonstrate "retention" require an animal to return to places most recently visited.

\section{Method}

The 10 rats used in the preceding three experiments served as subjects. A single eight-arm maze from the set used in Experiment 1 was placed in Room 1 and served as the only maze used in this experiment.

The experiment was carried out over a period of 16 days, which consisted of four blocks of 4 days each. "Different," "random," "same," and control conditions each were tested once within a block of days, and the orders in which these conditions were tested were randomized between subjects and blocks of days.

At the beginning of a trial, a rat was allowed to enter four randomly chosen alleys on the maze and to eat a pellet at the end of each arm. The rat then was placed at the end of four alleys in the "different," "random," and "same" conditions and was allowed to eat one pellet at the end of each alley. Placements consisted of the experimenter's gently holding the animal at the end of the alley for $10 \mathrm{sec}$ while the food pellet was consumed. As soon as the 10sec placement on one alley was completed, the rat was moved to the next alley for its 10-sec placement there. Depending on the condition being tested, placements were different from, the same as, or random with respect to the alleys entered on the initial forced choices. When the fourth placement was complete, the animal was returned to the central platform with all alleys open but only the alleys not entered on the initial forced choices containing a food pellet. The animal was allowed to choose alleys freely until all four food pellets had been acquired. On control trials, an animal was detained in a cage in the experimental room for $\mathbf{4 0} \mathrm{sec}$ between the initial forced choices and the retention test; the animal was allowed to consume four food pellets during the retention interval.

\section{Results}

The results are shown in the third row of Table 1. The mean percentages of correct choices indicate that 
the "same" and control conditions were substantially higher than the "random" and particularly the "different" conditions. A conditions $\times$ subjects analysis of variance showed that conditions was a significant effect $[F(3,27)=6.58, p<.01]$. A NewmanKeuls test comparing individual means with one another revealed significant differences between the "different" and control conditions, between the "different" and "same" conditions, and between the "random" and "same" conditions. No other differences between means were found to be significant.

The outcome of this experiment indicates that RI can be produced when a single maze is used, if the interpolated events involve placement at exactly those positions in space to which the rat must return in order to show retention of the initial forced alley entrances. These findings contrast with those of Experiments $2 a$ and $2 b$, in which displacement of Maze 1 and the interpolated maze either horizontally or vertically yielded no evidence of RI.

It is interesting to note that although RI was found in the "different" condition, performance was still fairly accurate in this condition $\mathbf{7 2 . 5 \%}$ ). If it were hypothesized that rats would avoid the alleys in which they were recently placed, choice of these alleys should approach $0 \%$. Another hypothesis is that rats in the RI conditons would choose randomly among the eight alleys, without entering the same alley twice on the first four choices. However, performance in the "different" condition significantly exceeded the $50 \%$ level expected by chance $(p<.01)$. The fact that animals in the "different" condition scored substantially higher than chance suggests that, although placement on different alleys caused some interference, animals were able to discriminate to a certain extent between initial forced choices and interpolated direct placements.

\section{Discussion}

Taken individually, these experiments suggest that RI was produced only when initial and interpolated visits to the end of arms occurred on the same maze, since the relationship between initial alleys visited and interpolated alleys visited was a significant factor only in Experiment $2 c$. Although Experiments $2 a$ and $2 \mathrm{~b}$ indicate little difference between the "different," "random," and control conditions, there is a suggestion in both experiments that the "same" condition is superior to the others. In order to evaluate this possibility further, the data from all three experiments were analyzed together within an experiments $\times$ conditions $x$ subjects analysis of variance. Significant effects were found for both experiments $[F(2,18)=$ 4.55, $\mathrm{p}<.05]$ and conditions $[\mathrm{F}(3,27)=4.41, \mathrm{p}<.01]$, but the experiments $\times$ conditions interaction was not significant $[F(6,54)=1.12, p>.05]$. A NewmanKeuls test showed that the effect of experiments re- sulted from significantly higher performance in Experiment $2 \mathrm{a}(\mathbf{8 7 . 3 \%})$ than in Experiments $2 \mathrm{~b}(\mathbf{7 9 . 2 \%})$ and $2 \mathrm{c}(80.0 \%)$, which did not differ from one another. The reason for this difference between experiments is not clear. When averaged across experiments, the mean percentages of correct choices for conditions "different," "random," control, and "same" were $78.6 \%, 80.3 \%, 82.3 \%$, and $87.4 \%$, respectively. A Newman-Keuls test indicated that the "same" condition was significantly higher than the "different" and "random" conditions but that no other pair of means differed significantly.

This analysis of the data from all three experiments combined suggests that there was some influence of the relationship of initial and interpolated arm visits in Experiments $2 a$ and $2 b$, as well as in Experiment $2 c$. However, the effect appears to be limited to the beneficial consequences of the "same" condition. While the "same" condition exceeded the "different" and "random" conditions, there was no indication that these latter two conditions differed from the control condition. This pattern of results indicates that allowing the rat to visit alleys pointing in the same direction on displaced mazes may have facilitated correct choices on the retention test but that conflicting forced choices did not inhibit correct choices, when performance is compared with the control condition in which no interpolated choices were given. An examination of the percentages in Table 1 clearly supports this position, since there is very little difference among the "different," "random," and control conditions in Experiments $2 \mathrm{a}$ and $2 b$. Only in Experiment $2 c$ do we find evidence that the "different" condition had an inhibitory effect, relative to the control condition. It would appear that the relationship between arms visited on initial and interpolated mazes had some facilitatory effect in the "same" conditions in Experiments $2 a$ and $2 b$ but was a more powerful inhibitory manipulation in Experiment 2c.

\section{GENERAL DISCUSSION}

An overall view of the experiments reported here indicates that $\mathbf{R I}$ in rat spatial memory can be demonstrated under certain circumstances and not others. Beatty and Shavalia (1980b) and Maki et al. (1979) demonstrated that RI for a series of forced choices on the eight-arm maze could not be achieved by a variety of sources of sensory stimulation or by actually entering alleys on another maze in a different context. These experiments extend those observations. In agreement with Beatty and Shavalia and Maki et al., Experiment 1 showed that RI could not be demonstrated with 1 and 2 interpolated mazes, even when the contexts in which Maze 1 and the interpolated mazes were placed were similar. Only 
when the task required the rat to remember alleys entered on three interpolated mazes did RI appear. It appears that the rat's spatial memory is surprisingly resistant to $R I$ but can be made to demonstrate RI when sufficiently overloaded with interpolated information. The fact that retention declined progressively as the number of mazes input or the ordinal position of the retention test increased (Figure 1) suggests that the spatial memory system may become further overburdened and interfered with as sources of PI and output interference increase.

Experiments $2 a$ and $2 b$ demonstrate further evidence of resistance to RI. These experiments used only one interpolated maze and were designed to maximize the conditions for the discovery of RI by (1) using exactly the same context (room) for initial and interpolated events, (2) having corresponding maze arms pointing in exactly the same direction and hence at the same extramaze cues, and (3) using an experimental design in which "different," "random," "same," and control conditions should provide a sensitive test of RI. The important factor that differentiated Maze 1 and interpolated maze experiences was the displacement of the mazes in space, horizontally in Experiment $2 \mathrm{a}$ and vertically in Experiment 2b. Since no RI was found in these experiments, it appears that perception of similar sets of extramaze cues from somewhat different positions allowed rats to encode Maze 1 and interpolated maze alley entrances as easily discriminated memories. Support for this interpretation was found in Experiment 2c, in which RI was produced when interpolated events involved direct placement at exactly the positions to which animals must return in order to enter a correct alley on the retention test. However, performance in the "same" condition in Experiments $2 a$ and $2 b$ suggested retroactive facilitation, and the overall analysis of Experiments $2 a, 2 b$, and $2 \mathrm{c}$ supports this observations. It may be that rats can readily discriminate between Maze 1 and interpolated maze experiences when these experiences conflict with one another but can make beneficial use of information from two places when the experiences dictate the same behavior.

These results may give us some hints about the way in which rats encode alley entrances on the radial maze. The finding that rats showed no RI with mazes separated horizontally or vertically within the same general context suggests that the rat is able to encode very precisely its position in space. Although arms on two mazes may point at the same cues, as long as the rat can experience these cues from different perspectives, different locations in space will be encoded. This might not be expected if the only cues an animal were using to remember alleys entered were gross features of the environment, such as brightness of a wall or the presence of a door. The rat may be able to combine such extramaze information with information about distance from cues and the angle from which cues are viewed to allow a precise specification of a point in space.

The fact that RI was found in Experiment 2c, which involved direct placements on the same maze as the forced choices and retention test, agrees well with the finding of PI in rat spatial memory by Roberts and Dale (1981). In the Roberts and Dale experiments, PI was found when rats were given repeated massed tests on the same maze. It was argued that PI arose from a failure to discriminate how recently alleys on the maze had been entered. That is, memories of entrances into alleys on Trial $n-1$ would be difficult for the animal to discriminate from memories of entrances and nonentrances into alleys on Trial $n$. The important point for the present discussion is that PI arises partially because alleys experienced on Trial $n-1$ and Trial $n$ are experienced at exactly the same positions in space and hence are encoded in the same way. Only the point in time at which these alleys were experiences would serve as a cue for discrimination between memories. The argument advanced here, then, is that a critical factor in both RI and PI in spatial memory is the use of to-beremembered and interfering events that involve travel to or placement at identical points in space. An interesting prediction follows from this analysis. Since RI seems to be eliminated by separating the initial and interpolated mazes in space, PI should be similarly affected by this manipulation. The prediction is that rats given repeated trials on the same maze, but with the maze moved from one part of a room to another on each trial, should show no PI.

The arguments presented here on the basis of these experiments seem to articulate well with the recently rekindled interest in Tolman's concept of a cognitive map (Menzel, 1978; Nadel \& Willner, 1980; O'Keefe \& Conway, 1980; O'Keefe \& Nadel, 1978; Olton, 1977; Roberts, 1979). According to the notion of a cognitive or spatial map, animals learn very rapidly about the places of objects in their environment and the relationships of these objects to one another. This map then is used to locomote through the environment to places where food has previously been found and to avoid places where the food supply has been exhausted. Although the objects in its environment may remain constant relative to one another, the position of the organism relative to these objects may be changing constantly. Changes in an animal's distance from objects and the perspective from which it views them allows the animal to constantly keep track of its own position. What is suggested here is that a rat not only forms a map of the environment in which it is placed, but also establishes a very accurate memory of the exact places to which it has traveled in that environment. Although the rat may perceive the same 
general set of cues from the ends of the arms of two mazes that are pointed in the same direction, a separation of these arms either vertically or horizontally allows the animal to encode these experiences as memories of quite different places.

\section{REFERENCES}

Beatty, W. W., \& Shavalia, D. A. Spatial memory in rats: Time course of working memory and effect of anesthetics. Behavioral and Neural Biology, 1980, 28, 454-462. (a)

Beatty, W. W., \& Shavalia, D. A. Rat spatial memory: Resistance to retroactive interference at long retention intervals. Animal Learning \& Behavior, 1980, 8, 550-552. (b)

Chiszar, D. A., \& Spe ar, N. E. Stimulus change, reversal learning, and retention in the rat. Journal of Comparative and Physiological Psychology, 1969, 69, 190-195.

Cook, R. G. Retroactive interference in pigeon short-term memory by a reduction in ambient illumination. Journal of Experimental Psychology: Animal Behavior Processes, 1980, 6, 326-338.

Crowder, R. G. Proactive and retroactive inhibition in the retention of a T-maze habit in rats. Journal of Experimental Psychology, 1967, 74, 167-171.

D'Amato, M. R., \& O'Neill, W. Effect of delay-interval illumination on matching behavior in the capuchin monkey. Journal of the Experimental Analysis of Behavior, 1971, 15, 327-333.

Frankmann, J. P. Effect of amount of interpolated learning and time interval before test on retention in rats. Journal of Experimental Psychology, 1957, 54, 462-466.

Grant, D. S., \& Roberts, W. A. Sources of retroactive inhibition in pigeon short-term memory. Journal of Experimental Psychology: Animal Behavior Processes, 1976, 2, 1-16.

Ho, Y. H. Transfer and degree of integration. Journal of Comparative Psychology, 1928, 8, 87-99.

Maki, W. S., Brokofsky, S., \& Berg, B. Spatial memory in rats: Resistance to retroactive interference. Animal Learning \& Behavior, 1979, 7, 25-30.
Marx, M. H. The effects of cumulative training upon retroactive inhibition and transfer. Comparative Psychology Monographs, $1944,18,1-62$.

Menzel, E. W. Cognitive mapping in chimpanzees. In $\mathbf{S}$. $\mathrm{H}$. Hulse, H. Fowler, \& W. K. Honig (Eds.), Cognitive processes in animal behavior. Hillsdale, N.J: Erlbaum, 1978.

Nadel, L., \& Willner, J. Context and conditioning: A place for space. Physiological Psychology, 1980, 8, 218-228.

O'KeEfe, J., \& Conway, D. H. On the trail of the hippocampal engram. Physiological Psychology, 1980, 8, 229-238.

O'KeEFE, J., \& NADEL, L. The hippocampus as a cognitive map. Oxford: Clarendon Press, 1978.

Olton, D. S. Spatial memory. Scientific American, 1977, 236, 82-98.

Olton, D. S. Characteristics of spatial memory. In S. H. Hulse, H. Fowler, \& W. K. Honig (Eds.), Cognitive processes in animal behavior. Hillsdale, N.J: Erlbaum, 1978.

Olton, D. S. Mazes, maps, and memory. American Psychologist, $1979,34,588-596$.

Olton, D. S., Collison, C., \& Werz, M. A. Spatial memory and radial arm maze performance of rats. Learning and Motivation, 1977, 8, 289-314.

Roberts, W. A. Spatial memory in the rat on a hierarchical maze. Learning and Motivation, 1979, 10, 117-140.

Roberts, W. A., \& DAle, R. H. I. Remembrance of places lasts: Proactive inhibition and patterns of choice in rat spatial memory. Learning and Motivation, 1981, 12, 261-281.

Tranberg, D. K., \& Rilling, M. Delay-interval illumination changes interfere with pigeon short-term memory. Journal of the Experimental Analysis of Behavior, 1980, 33, 39-49.

Walker, J. A., \& Olton, D. S. The role of response and reward in spatial memory. Learning and Motivation, 1979, 10, 73-84.

Waters, R. H., \& Vitale, A. G. Degree of interpolated learning and retroactive inhibition in maze learning. Journal of Comparative Psychology, 1945, 38, 119-126.

Zentall, T. R. Effects of context change on forgetting in rats. Journal of Experimental Psychology, 1970, 86, 440-448.

(Manuscript received January 28, 1981; revision accepted for publication July 23,1981 .) 